\title{
RETRACTED ARTICLE: Application of embedded computer and improved genetic algorithm in the strategy of community of human destiny: the development of artificial intelligence in the context of Covid-19
}

\author{
Yaoyao Liu ${ }^{1} \cdot$ Yixin Zhang $^{1,2} \cdot$ Xiaoya Liu $^{3}$
}

Received: 22 January 2021 / Accepted: 25 March 2021 / Published online: 9 April 2021

(c) The Author(s), under exclusive licence to Springer-Verlag GmbH Germany, part of Springer Nature 2021

The Editor-in-Chief and the publisher have retracted this article. The article was submitted to be part of a guest-edited issue. An investigation by the publisher found a number of articles, including this one, with a number of concerns, including but not limited to compromised editorial handling and peer review process, inappropriate or irrelevant references or not being in scope of the journal or guest-edited issue. Based on the investigation's findings the Editor-inChief therefore no longer has confidence in the results and conclusions of this article.

Author Yaoyao Liu disagrees with this retraction. The Publisher has not been able to obtain a current email address for authors Yixin Zhang and Xiaoya Liu.
The online version of this article contains the full text of the retracted article as Supplementary Information.

Supplementary Information The online version contains supplementary material available at https://doi.org/10.1007/s12652-021-03218-5.

Publisher's Note Springer Nature remains neutral with regard to jurisdictional claims in published maps and institutional affiliations.

Springer Nature or its licensor (e.g. a society or other partner) holds exclusive rights to this article under a publishing agreement with the author(s) or other rightsholder(s); author self-archiving of the accepted manuscript version of this article is solely governed by the terms of such publishing agreement and applicable law.

Yaoyao Liu

zkk6180@163.com

1 School of Ethnology and Sociology, Minzu University of China, Beijing 100081, China

2 Institute of Pharmacology and Toxicology, Academy of Military Medical Sciences, Beijing 100039, China

3 College of Pharmaceutical Science, Shandong University of Traditional Chinese Medicine, Shandong 250355, Jinan, China 